\title{
The non-perturbative BRST quartet mechanism in Landau gauge QCD: Ghost-gluon and ghost-quark bound states
}

\author{
Natalia Alkofer* \\ Institut für Physik, Karl-Franzens-Universität, Universitätsplatz, 5, A-8010 Graz, Austria \\ E-mail: natalia.alkofereedu.uni-graz.at \\ Reinhard Alkofer \\ Institut für Physik, Karl-Franzens-Universität, Universitätsplatz, 5, A-8010 Graz, Austria \\ E-mail: reinhard.alkofer@uni-graz.at
}

\begin{abstract}
The non-perturbative BRST quartet mechanism in infrared Landau gauge QCD is presented. It is demonstrated that positivity violation for transverse gluons allows to identify the gluon's nonperturbative BRST quartet. To describe the respective BRST-daughter state a truncated BetheSalpeter equation for the gluon-ghost bound state is investigated. An analogous construction for quarks yields a truncated Bethe-Salpeter equation for the quark-ghost bound state. The gluonghost bound state equation in two space-time dimensions has been numerically solved.
\end{abstract}

Xth Quark Confinement and the Hadron Spectrum

8-12 October 2012

TUM Campus Garching, Munich, Germany

\footnotetext{
* Speaker.
} 
The Landau gauge gluon propagator violates positivity, see e.g. Refs. [1] and references therein. Due to this property the one-(transverse)gluon-state possesses a negative norm and is thus removed from the $S$-matrix. This construction can be formalised by considering the BRST cohomology but this does not tell us how the removal of BRST non-invariant states occurs dynamically. Within the context of Landau gauge QCD we formulated some first steps into this direction [2]. The non-perturbative character of the BRST quartets of the transverse gluon (quark) is evident from the fact that they contain besides the transverse gluon (quark) only bound states. Therefore we need a method to solve bound state equations in an approximately symmetry-preserving way.

The quantum state of one transverse gluon constitutes a parent state in its BRST quartet, a fact which allows to identify the other members. The role of these bound states in covariantly gaugefixed Yang-Mills (YM) theory as well as for the kinematical aspects of gluon confinement can then be elucidated based on purely algebraic considerations. For quarks we adopt a similar approach as for the gluons in order to clarify by constructing a (hypothetical) quark BRST quartet whether quarks are also positivity violating.

The BRST transformation $\delta_{B}$ is defined as:

$$
\delta_{B} A_{\mu}^{a}=\widetilde{Z}_{3} D_{\mu}^{a b} c^{b} \lambda, \delta_{B} q=-i g t^{a} \widetilde{Z}_{1} c^{a} q \lambda, \delta_{B} c^{a}=-\frac{g}{2} f^{a b c} \widetilde{Z}_{1} c^{b} c^{c} \lambda, \delta_{B} \bar{c}^{a}=B^{a} \lambda, \delta_{B} B^{a}=0
$$

where $D_{\mu}^{a b}$ is the covariant derivative. The parameter $\lambda$ has ghost number $N_{\mathrm{FP}}=-1$, and $B^{a}$ is the Nakanishi-Lautrup field. $\widetilde{Z}_{1}$ and $\widetilde{Z}_{3}$ are the ghost-gluon-vertex and the ghost wave function renormalisation constants. Following the usual notation the starting (negative norm) state is called 1st parent, its BRST transform 1st daughter. The Faddeev-Popov conjugated state of the latter is named 2nd parent, the corresponding BRST transform 2nd daughter. Eq. (1) allows to conclude that in the respective BRST quartet the daughter state of the transverse gluon is a gluon-ghost bound state [2]. The second parent is a gluon-antighost bound state with its BRST transform being the second daughter state. This algebraic structure guarantees the vanishing of $S$-matrix elements between transverse gluons and BRST singlets, the latter being the physical colour-neutral states.

There is no definite result on positivity for quarks. Within functional methods this is due to the sensitivity of the analytic structure of the quark propagator to details of the quark-gluon vertex [3]. This vertex is, on the other hand, also very strongly influenced by dynamical and/or explicit chiral symmetry breaking [4]. Therefore the resulting dynamical quark mass generation is highly susceptible to details of the dynamics. Which mechanism secures then that the BRST-quartet ghostquark bound states are degenerate with the quark states is completely unknown. This motivates to investigate the bound state equations for the non-perturbative BRST quartet states with the aim to gain insight into the underlying mechanisms.

In Ref. [2] we derived the Bethe-Salpeter equations for ghost-gluon and ghost-quark bound states in a truncation scheme based on an infrared analysis. The gluon-ghost equation is graphically represented in Fig. 1, and in the following we will concentrate on this equation. One notices that the gluon-ghost Bethe-Salpeter equation contains two terms, a ghost exchange and a gluon exchange. Both are of the same infrared order because in the so-called scaling solution (for details of its properties see Ref. [5] and references therein) the fully dressed three-gluon vertex is infrared divergent [6]. Employing the propagator parametrisations of e.g. Ref. [3], the ghost-gluon vertex of Ref. [7], and the three-gluon vertex of Ref. [8], one arrives at a self-consistent equation for the 

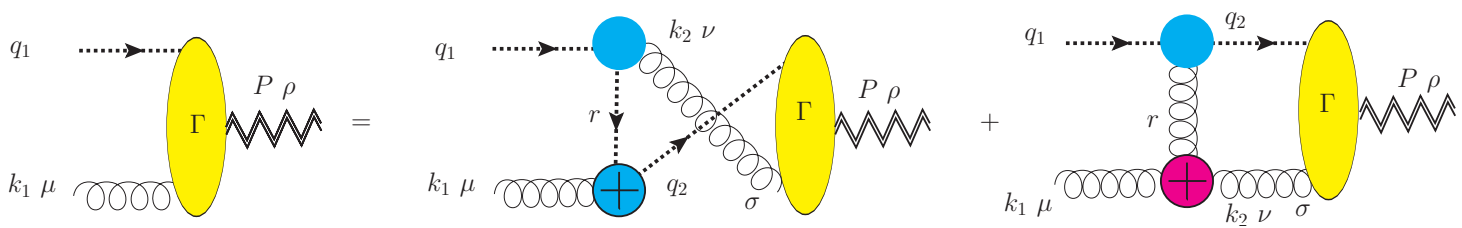

Figure 1: Representation of the ghost-gluon Bethe-Salpeter equation. Crosses denote dressed vertices.

gluon-ghost Bethe-Salpeter amplitude with only known quantities as input. As demonstrated in Ref. [2], this bound state in the adjoint colour representation is transverse, i.e., it is as expected in the massless representation of the Poincare group. In the limit of soft momenta the ghost-gluon bound state is then described by one amplitude $F\left(q^{2}\right)$ with $q$ being the relative momentum between the gluon and the ghost. A restriction to the presumably dominant ghost exchange and taking into account that the ghost-gluon vertex can be accurately approximated by the bare vertex leads to a linear integral equation for $F\left(q^{2}\right)$ with only the gluon and ghost dressing functions, $Z\left(k^{2}\right)$ and $G\left(k^{2}\right)$, respectively, as input [2].

As recently propagator and vertex functions for two space-time dimensions became available [9] we solved the ghost-gluon equation in two dimensions,

$$
F\left(k_{1}^{2}\right)=k_{1}^{2} Z_{3}^{2} g^{2} N_{c}^{2} \int \frac{d^{2} k_{2}}{(2 \pi)^{2}} \frac{G\left(\left(k_{1}+k_{2}\right)^{2}\right)}{\left(k_{1}+k_{2}\right)^{2}} \frac{G\left(k_{2}^{2}\right)}{k_{2}^{2}} \frac{Z\left(k_{2}^{2}\right)}{k_{2}^{2}}\left(1-\frac{\left(k_{1} \cdot k_{2}\right)^{2}}{k_{1}^{2} k_{2}^{2}}\right) F\left(k_{2}^{2}\right),
$$

and provided evidence for the existence of this bound state. Details will be published elsewhere.

\section{Acknowledgments}

We thank the organisers of the Xth Quark Confinement and the Hadron Spectrum conference for all their efforts which made this extraordinary event possible. N.A. gratefully acknowledges financial support by the conference. We thank M. Q. Huber for helpful discussions.

\section{References}

[1] P. O. Bowman et al., Phys. Rev. D 76 (2007) 094505 [arXiv:hep-lat/0703022]; S. Strauss, C. S. Fischer and C. Kellermann, Phys. Rev. Lett. 109 (2012) 252001 [arXiv:1208.6239 [hep-ph]].

[2] N. Alkofer and R. Alkofer, Phys. Lett. B702 (2011) 158[arXiv:1102.2753 [hep-th]]; PoS FACESQCD (2011) 043 [arXiv:1102.3119 [hep-th]]; PoS QCD-TNT-II (2011) 002 [arXiv:1112.4483 [hep-th]].

[3] R. Alkofer et al., Phys. Rev. D70 (2004) 014014 [hep-ph/0309077];

Nucl. Phys. Proc. Suppl. 141 (2005) 122 [hep-ph/0309078].

[4] R. Alkofer et al., Annals Phys. 324 (2009) 106 [arXiv: 0804.3042 [hep-ph]]; M. Hopfer, A. Windisch and R. Alkofer, PoS CONFINEMENTX (2013) 073 [arXiv:1301.3672 [hep-ph]].

[5] C.S. Fischer, A. Maas and J.M. Pawlowski, Ann. Phys. 324 (2009) 2408 [arXiv:0810.1987 [hep-ph]].

[6] R. Alkofer, C. S. Fischer, F. J. Llanes-Estrada, Phys. Lett. B 611 (2005) 279 [arXiv:hep-th/0412330].

[7] W. Schleifenbaum et al., Phys. Rev. D 72 (2005) 014017 [arXiv:hep-ph/0411052].

[8] R. Alkofer, M. Q. Huber, K. Schwenzer, Eur. Phys. J. C 62 (2009) 761 [arXiv:0812.4045 [hep-ph]].

[9] M. Q. Huber, A. Maas and L. von Smekal, JHEP 1211 (2012) 035 [arXiv:1207.0222 [hep-th]]. 